\title{
Effects of 'Thickness on the Residual Stress Behavior of High Temperature Polyimide Films
}

\author{
Hyunsoo ChUnG, Yung-Il JoE, and Haksoo HaN ${ }^{\dagger}$ \\ Department of Chemical Engineering, Yonsei University \\ 134 Shinchon-Dong, Seodaemun-Gu, Seoul 120-749, Korea
}

(Received July 7, 1999)

\begin{abstract}
The effects of thickness on the residual stress behavior of the hinged structure poly(4,4oxydiphenylene pyromellitimide) (PMDA-ODA) and rigid planar structure poly ( $p$-phenylene biphenyltetracarboximide) (BPDA-PDA) polyimide were studied. Depending on the polyimide chemistry, residual stress behavior of polyimide film with thickness variation was significantly different. For fully cured BPDA-PDA polyimide at $400^{\circ} \mathrm{C}$, residual stress of polyimide film increased from 5.4 MPa to $45 \mathrm{MPa}$. For fully cured PMDA-ODA polyimide at $400^{\circ} \mathrm{C}$, residual stress of polyimide film with thickness variation changed from 29.1 MPa to 40.2 MPa. The effect of thickness on the residual stress for the rigid structure BPDA-PDA was significant, but relatively not for the semiflexible structure PMDA-ODA. Residual stress behavior was quite related to the morphology of fully cured polyimide as shown WAXD analysis. Higher molecular anisotropy leads to lower residual stress of polyimide film. Residual stress behavior of polyimide films with thickness variation is thus closely related to chain rigidity and chain anisotropy associated with polyimide chemistry and morphological structure.
\end{abstract}

KEY WORDS Residual Stress / Film Thickness / Morphology / Molecular Anisotropy /

Polyimides have many applications for their excellent thermal, mechanical, and electrical properties. In multilevel interconnection packaging, polyimides seem very good candidates as dielectric materials. ${ }^{1-3}$ In these applications, one major concern about the use of polyimides is residual stress including dimensional stability. To minimize residual stress induced by thermal mismatch, an appropriated polyimide, which has a thermal expansion coefficient (TEC) matching that of the desired substrate, is recommended between the layers to insure long-term device reliability such as displacement, crack, and delamination. TEC of polyimide is a function of backbone chemistry and morphology and is strongly influenced by molecular orientation. To some extent, TEC is influenced by crystallinity (molecular ordering). Understanding the relationship between molecular orientation and residual stress properties is therefore of considerable interest. Another interesting subject in residual stress is that an appropriate coating thickness should be applied.

Minimizing stress during device fabrication has been the primary concern of the electronics industry and many initial studies focusing on structure development monitored stress levels during cure. ${ }^{4-7}$ This is because the applied polyimide films may have a serious thermal mismatch due to TEC different from that of substrate on thermal curing or thermal cycling. Elsner et al. ${ }^{8}$ observed that the final state of stress in the coatings was independent of the drying temperature and varied linearly with the final cure temperature. Han et al. ${ }^{9}$ studied the effect of heating rate during cure on the final state of stress in fully cured spin coating and found that the stress increased with heating rate. Bauer and Farris ${ }^{10}$ investigated the development of stresses in poly(amic acid)s during solvent removal related not to the initial solution concentration but to the concentration at which the material could first sustain a load. Researchers have recently focused on morphology changes during cure and their impact on residual stress of the resulting film of coating. ${ }^{11-15,28,29}$ Jou et al. ${ }^{11-13}$ have reported that the effect of curing temperature on the TEC and morphology of polyimides of $6-27 \mu \mathrm{m}$ thickness significantly influenced on residual stress, examined using the bending beam method. Ree et al. observed the residual stress of the films of $10-100 \mu \mathrm{m}$ thickness increased with film thickness, whereas the anisotropy of the refractive indices decreased with it. ${ }^{14}$ The degree of in-plane orientation in the spin-cast films is suspected to depend on curing conditions, solvent systems, and polyimide chain characteristics. The degree of chain orientation in polyimide films may vary significantly with thickness. ${ }^{15}$

However, the relationship of residual stress with morphology (molecular orientation, molecular chain order) is not fully understood in thermally imidized films in spite of numerous studies performed on residual stress in polyimides. Therefore, the effect of thickness on residual stress and molecular anisotropy of polyimide films cured on a substrate were investigated for two different polyimides. To quantify the interaction of thermal mismatch with the polyimide structures depending on various thicknesses, residual stress experiments were carried over a processing temperature range of $25-400^{\circ} \mathrm{C}$ by using in situ thin film stress analyzer (TFSA). The effect of thickness on the morphology of resulting film was analyzed by wide-angle X-ray diffraction (WAXD).

\section{EXPERIMENTAL}

\section{Materials and Film Preparation}

Poly(amic acid)s (PAAs) were synthesized in dry $N$ methyl-2-pyrrolidone (NMP) from the respective dianhydrides and diamines; poly (4,4-oxydiphenylene pyro-

\footnotetext{
${ }_{{ }^{\dagger}}$ To whom correspondence should be addressed (Tel : +82-2-361-2764, Fax : +82-2-312-6401, e-mail : hshan@bubble.yonsei.ac.kr).
} 


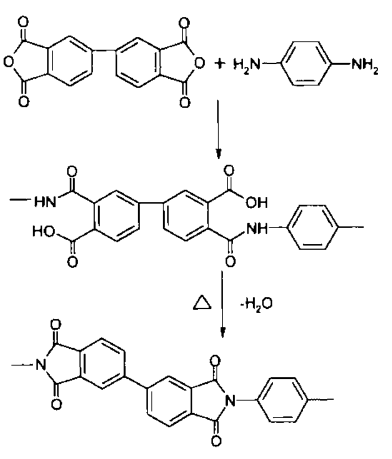

BPDA-PDA

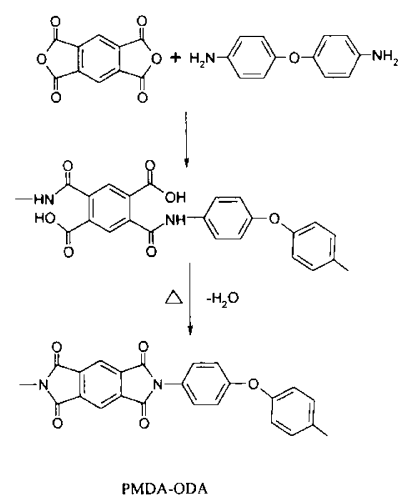

PMDA-ODA
Figure 1. Synthetic scheme and chemical structure of polyimide in this study.

mellitamic acid) (PMDA-ODA PAA) and $\operatorname{poly}(p-$ phenylene biphenyltetracarboxamic acid) (BPDA-PDA PAA $)^{1-3}$ as shown in Figure 1. In the synthesis of poly(amic acid)s, attempt to control molecular weights was made by creating a stoichiometric imbalance of the monomers using small excess diamine. ${ }^{2,3}$ Intrinsic viscosity was measured at NMP to be $0.65 \mathrm{dL} \mathrm{g}^{-1}$ for BPDA-PDA PAA and $0.62 \mathrm{dL} \mathrm{g}^{-1}$ for PMDA-ODA PAA. The concentrations of PMDA-ODA and BPDA-PDA precursor were $15 \mathrm{wt} \%$ in NMP. The films of precursor were prepared by spin coating and soft baked at $80^{\circ} \mathrm{C}$ for 30 min in a convection oven.

Polyimide films (PMDA-ODA and BPDA-PDA) were finally prepared in the thickness range of $8-130 \mu \mathrm{m}$. Here, film thickness was measured using an alphastepper (Tencor Instruments Co.). For the polyimide thick films $>30 \mu \mathrm{m}$, the thickness was controlled using the multispin coating on a silicon wafer followed by $80^{\circ} \mathrm{C}$ bake resulted in a $10-15 \mu \mathrm{m}$ film.

\section{Measurement}

The imidization of polyamic acid precursor on the $\mathrm{Si}$ substrate was performed with a one-step heating process up to $400^{\circ} \mathrm{C}$ with ramping rate of $2.0^{\circ} \mathrm{C} \mathrm{min}$ min $^{-1}$ and an nealing at $400^{\circ} \mathrm{C}$ for $1 \mathrm{~h}$. The residual stress behavior of polyimide films during thermal curing process was monitored geometrically by using TFSA as shown in Figure 2. To determine the residual stress from the measured radii of curvature a well known eq $1^{16}$ used. Residual stress was calculated from the measured radii of curvatures : $R_{1}$ and $R_{2}$.

$$
\sigma=\frac{E_{\mathrm{s}}}{6\left(1-\nu_{\mathrm{s}}\right)} \frac{t_{\mathrm{s}}^{2}}{t_{\mathrm{f}}}\left(\frac{1}{R_{2}}-\frac{1}{R_{1}}\right)
$$

$\sigma$ is residual stress in the polyimide film. The subscripts, $f$ and $s$ denote the polyimide film and substrate. $E, \nu$, and $t$ are Young's modulus, Poisson's ratio, and thickness of the substrate, respectively. $R_{1}$ and $R_{2}$ are wafer curvatures measured before and after film deposition. For $\mathrm{Si}(100)$ wafer, $E_{\mathrm{S}} /\left(1-\nu_{\mathrm{s}}\right)$ is $180500 \mathrm{MPa} .{ }^{17} \mathrm{In}-$ itial curvature of $\mathrm{Si}$ wafer and thickness of wafers were measured using TFSA and thickness gauge (SM 1201, Teclock Co., Japan), respectively. Residual stress behavior due to thermal history after cure was considered from final cure temperature to $50^{\circ} \mathrm{C}$ to exclude moisture

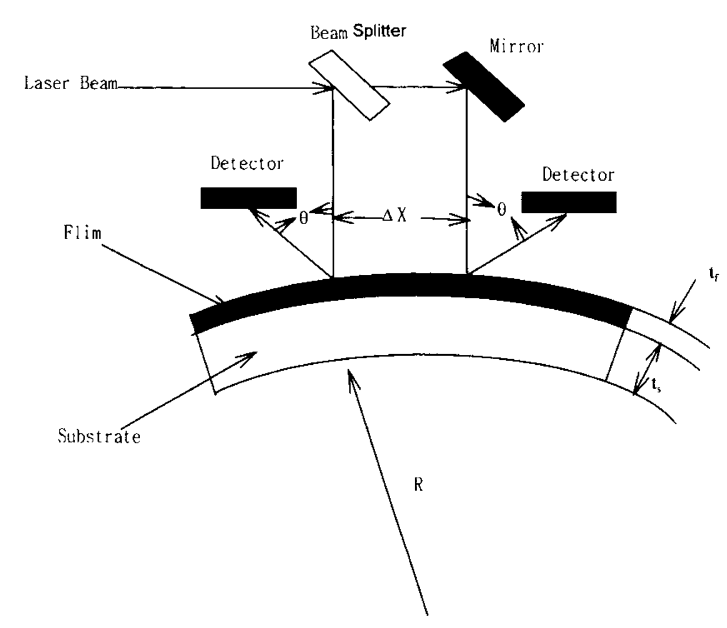

Figure 2. Schematic of thin film stress analyzer (TFSA).

effect on the polyimide films.

Wide-angle X-ray diffraction (WAXD) was used to examine morphological structures of polyimide films. WAXD patterns were collected using the $\theta-2 \theta$ method in the reflection and transmission mode over $2^{\circ}<2 \theta<$ $60^{\circ}$. A Rigaku CN 2155 powder diffractometer was used for these measurements at room temperature. Measured $\mathrm{X}$-ray diffraction intensity was corrected by the background run and normalized for the film samples by matching the integrated intensity over the range of 58$60^{\circ}(2 \theta){ }^{18,19}$ The $\mathrm{X}$-ray generator, $\mathrm{Cu}-K_{\alpha}$ radiation source $(\lambda=1.54 \AA)$ was run at $35 \mathrm{kV}$ and $40 \mathrm{~mA}$.

\section{RESULTS AND DISCUSSION}

The stress behavior of polyimide films was investigated with variation of thickness and different backbone polyimide precursors. During curing of poly(amic acid), volume shrinkage that accompanies the initial solvent evaporation at $80^{\circ} \mathrm{C}$ induces an initial stress in the poly(amic acid) film. ${ }^{20}$ As shown in Figures 3 , and 4, the initial stress of polyamic acid precursor decreased monotonically with increasing film thickness. The initial stress of BPDA-PDA and PMDA-ODA precursors was $5.5-17 \mathrm{MPa}$ for films of $<20 \mu \mathrm{m}$, whereas nearly $0 \mathrm{MPa}$ for thick film $>30 \mu \mathrm{m}$.

The stress of precursor film should be induced with imidization and solvent evaporation in precursor film during heating. However, residual stress of polyimide thin films $(<20 \mu \mathrm{m})$ continuously decreased with increasing temperature up to $250^{\circ} \mathrm{C}$ as shown in Figures 3 and 4. It may show that stress of polymer films was compensated by higher thermal mismatch between precursor and substrate. Residual stress of thick film $(>30 \mu \mathrm{m})$ was nearly zero in film before $175^{\circ} \mathrm{C}$. The relatively constant stress behavior probably may result from the plasticization of the polymer chains due to the presence of a large amount of solvent in precursor film. The effect of a large amount of solvent in the film would significantly lead to relatively low stress of thick films at initial stage of curing process. However, the stress of thick film $(>30$ $\mu \mathrm{m}$ ) increased from around $200^{\circ} \mathrm{C}$. The observed maximum stress at $200^{\circ} \mathrm{C}$ may result from the conversion of an amic acids group to imides group and solvent evapo- 


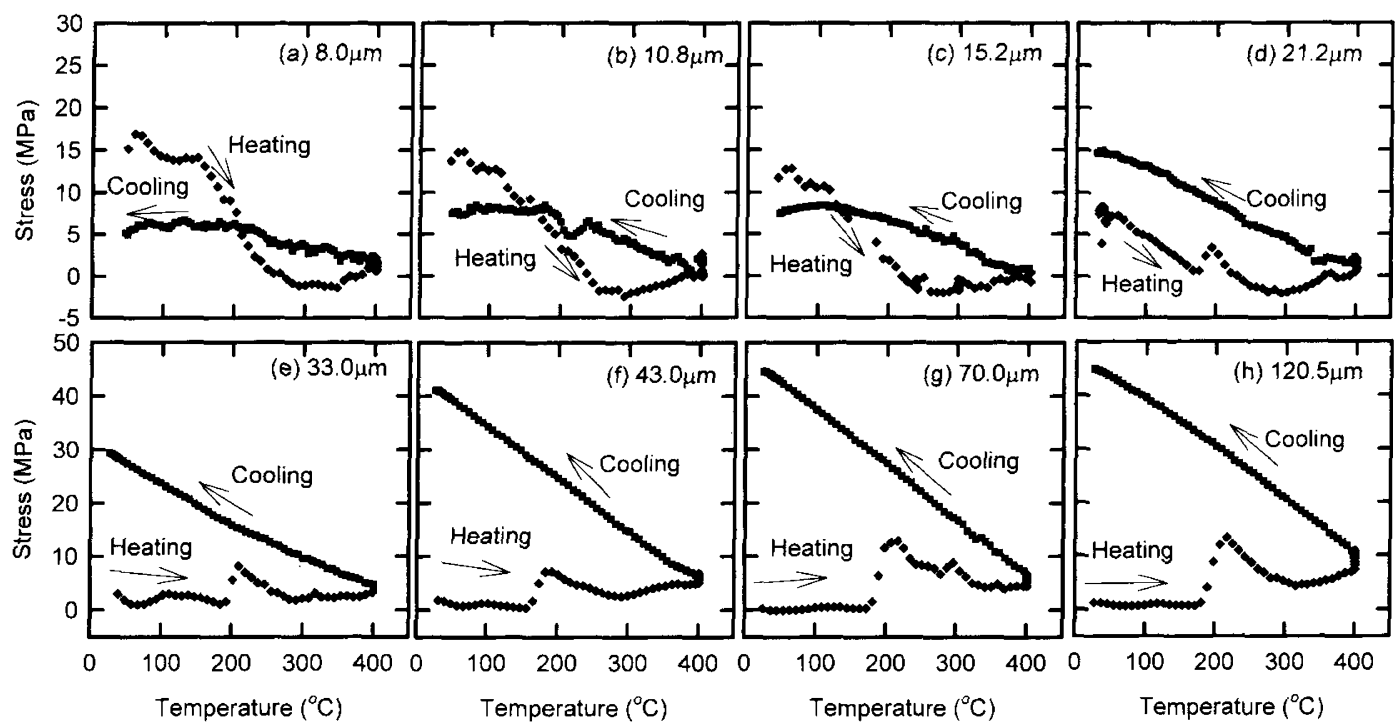

Figure 3. Dynamic stress behavior of BPDA-PDA polyimide films with various thicknesses.
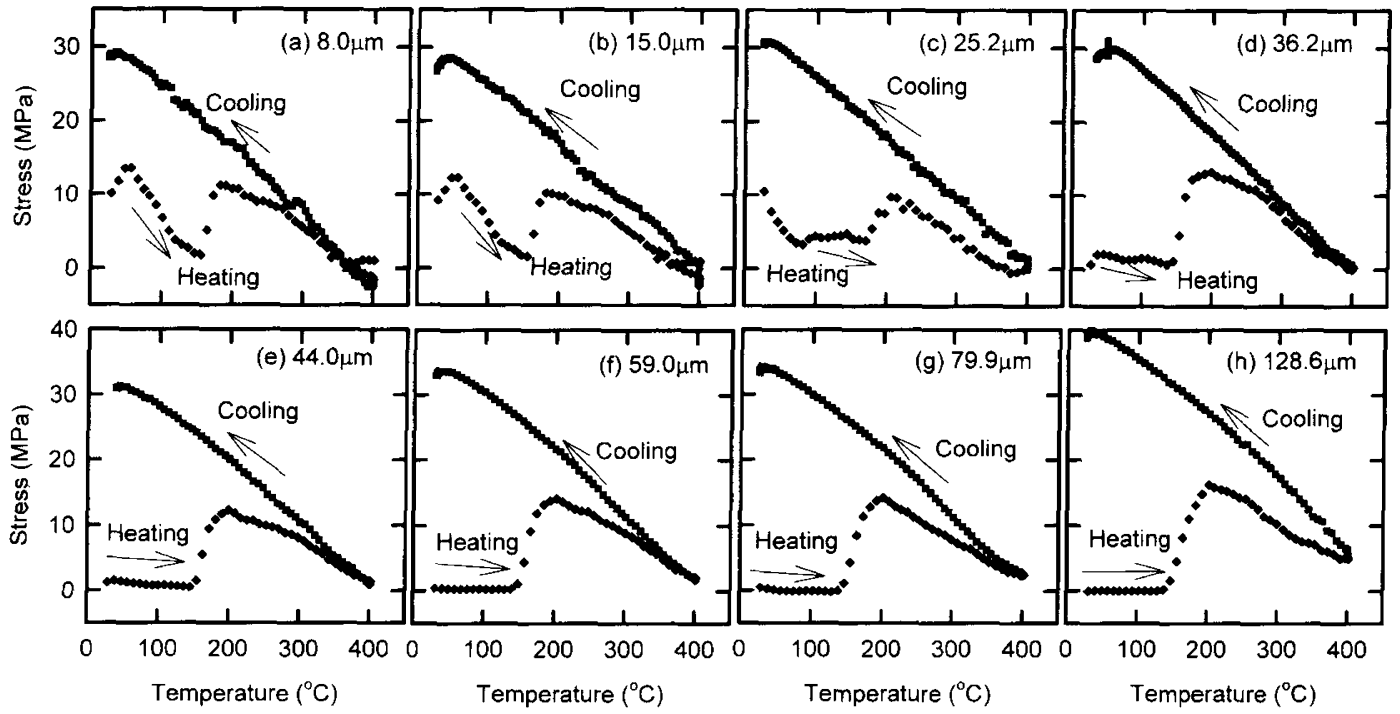

Figure 4. Dynamic stress behavior of PMDA-ODA polyimide films with various thicknesses.

ration (NMP $: \mathrm{bp} ; 202^{\circ} \mathrm{C}$ ). The stored stress decreased slightly with increasing temperature up to $300^{\circ} \mathrm{C}$, but nearly constant regardless of film thickness variation. Poly(amic acid)s precursor with high TEC converted to comparatively low TEC imide counterparts. The stress of BPDA-PDA films continuously slightly rises as temperature increases above $300^{\circ} \mathrm{C}$. The stress of BPDAPDA film does not descend to near zero at $400^{\circ} \mathrm{C}$. This may show the relatively high chain rigidity of BPDAPDA polyimide even at $400^{\circ} \mathrm{C}$.

For PMDA-ODA, stress behavior under heating process shows similar pattern to those of BPDA-PDA. However, the stress behavior of semi flexible PMDA-ODA films continuously descends with elevated temperature $\left(>300^{\circ} \mathrm{C}\right)$ after going through a maximum stress peak. The stress of PMDA-ODA film descends to near zero at $400^{\circ} \mathrm{C}$. This may indicate that the relatively low stress of PMDA-ODA at high temperature show more chain flexibility and mobility than the rigid BPDA-PDA.

The effect of thickness on residual stress of polyimide film after imidization for rigid structure BPDA-PDA and semiflexible structure PMDA-ODA is shown in Figure 5 and Table I. Residual stress of rigid BPDA-PDA polyimides changed from 5.4 to $45.0 \mathrm{MPa}$ depending on film thickness. Low residual stress of thin films is thus achieved only for the thin films $(<15 \mu \mathrm{m})$ and the characteristic properties of BPDA-PDA film stress such as film modulus and thermal expansion coefficient depend on the film thickness. Morphological structure of BPDAPDA polyimide may thus significantly changed due to thickness variation.

However, residual stress of PMDA-ODA polyimide after fully cured at $400^{\circ} \mathrm{C}$ was less sensitive to film thickness in comparison with that of BPDA-PDA polyimide. Residual stress of semiflexible PMDA-ODA polyimide film changed in the range $29-40 \mathrm{MPa}$ with thickness variation as shown in Figure 5. The change of morphology in flexible structure PMDA-ODA film may thus be much less sensitive to film thickness because they are nearly amorphous due to bendable ether linkage. The shrinking of spin-cast precursor films occurs only in the direction perpendicular to film plane, leading to in-plane 
H. Chung, Y.-I. JoE, and H. HAN

Table I. Residual stress of polyimide films with thickness variation ${ }^{2}$

\begin{tabular}{|c|c|c|c|c|}
\hline Backbone chemistry & $\frac{\text { Film thickness }}{\mu \mathrm{m}}$ & $\begin{array}{l}\text { Initial stress } \\
\quad\left(\text { at } 25^{\circ} \mathrm{C}\right)\end{array}$ & $\begin{array}{c}\text { Residual stress } \\
\left(\text { at } 400^{\circ} \mathrm{C}\right)\end{array}$ & $\begin{array}{c}\text { Residual stress } \\
\text { (at } 25^{\circ} \mathrm{C} \text { ) }\end{array}$ \\
\hline \multirow{9}{*}{ BPDA-PDA } & 8.0 & 17.0 & 1.2 & 5.4 \\
\hline & 10.8 & 15.5 & 2.5 & 7.7 \\
\hline & 15.2 & 14.0 & 2.2 & 8.5 \\
\hline & 21.2 & 8.1 & 2.5 & 15.0 \\
\hline & 33.0 & 5.5 & 5.1 & 29.8 \\
\hline & 43.0 & 1.1 & 6.3 & 41.2 \\
\hline & 64.1 & 0.8 & 7.5 & 41.0 \\
\hline & 70.1 & 1.0 & 8.1 & 43.5 \\
\hline & 120.5 & 0.5 & 10.5 & 45.0 \\
\hline \multirow{9}{*}{ PMDA-ODA } & 8.0 & 14.4 & 0 & 29.1 \\
\hline & 12.1 & 13.6 & 0 & 29.0 \\
\hline & 15.0 & 13.2 & 0 & 30.5 \\
\hline & 25.2 & 10.7 & 0 & 31.0 \\
\hline & 36.2 & 2.5 & 0.5 & 30.5 \\
\hline & 44.0 & 1.0 & 0.9 & 31.6 \\
\hline & 59.0 & 0.9 & 1.8 & 33.1 \\
\hline & 79.9 & 0.4 & 2.3 & 34.2 \\
\hline & 128.6 & 0.1 & 4.6 & 40.2 \\
\hline
\end{tabular}

${ }^{a}$ All residual stress experiments were run $25-400^{\circ} \mathrm{C}$.

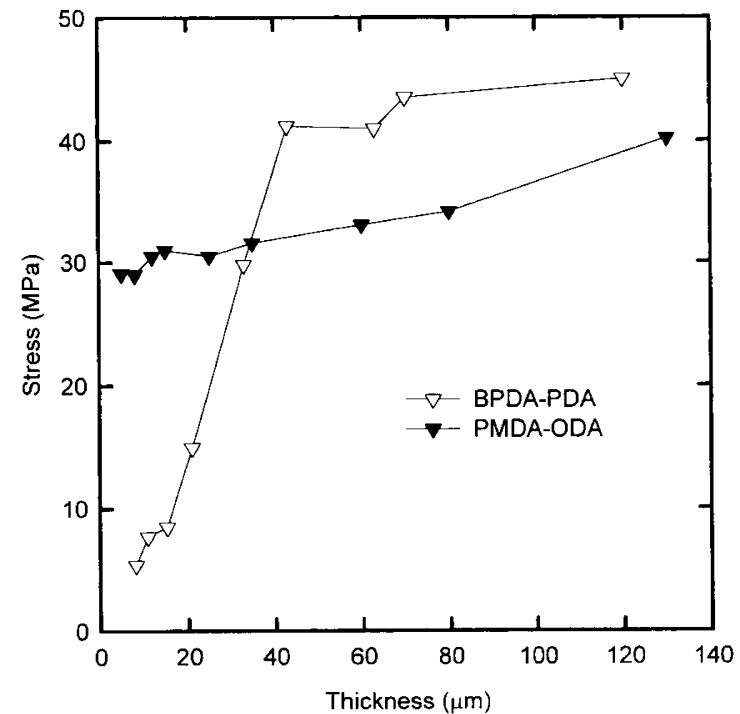

Figure 5. Effect of thickness on residual stress behavior of the rigid BPDA-PDA and PMDA-ODA films after fully cured at $400^{\circ} \mathrm{C}$.

orientation of polyimide chain ${ }^{19}$ during thermal imidization. This may affect the thermal expansivity and mechanical properties of film, which are related to the stress in the film after curing. This suggests that morphological structure such as chain orientation is significantly changed in BPDA-PDA film with increasing thickness above $15 \mu \mathrm{m}$, whereas relatively insensitive in PMDA-ODA film.

For BPDA-PDA films differing in thickness, the morphology was investigated using WAXD as shown in Figure 6. For the BPDA-PDA, the intensity of (004) diffraction peak in transmission pattern was significantly decreased with increasing thickness. This indicates that the degree of the molecular in-plane orientation was decreased in polyimide with increasing thickness. Coherence length from WAXD patterns of polyimide films was estimated as shown in Table II. The coherence length for the $(004)$ peak in BPDA-PDA decreased in the range of 105-122 $\AA$ depending upon film thickness. Coherence length is defined by Scherrer equation with the full

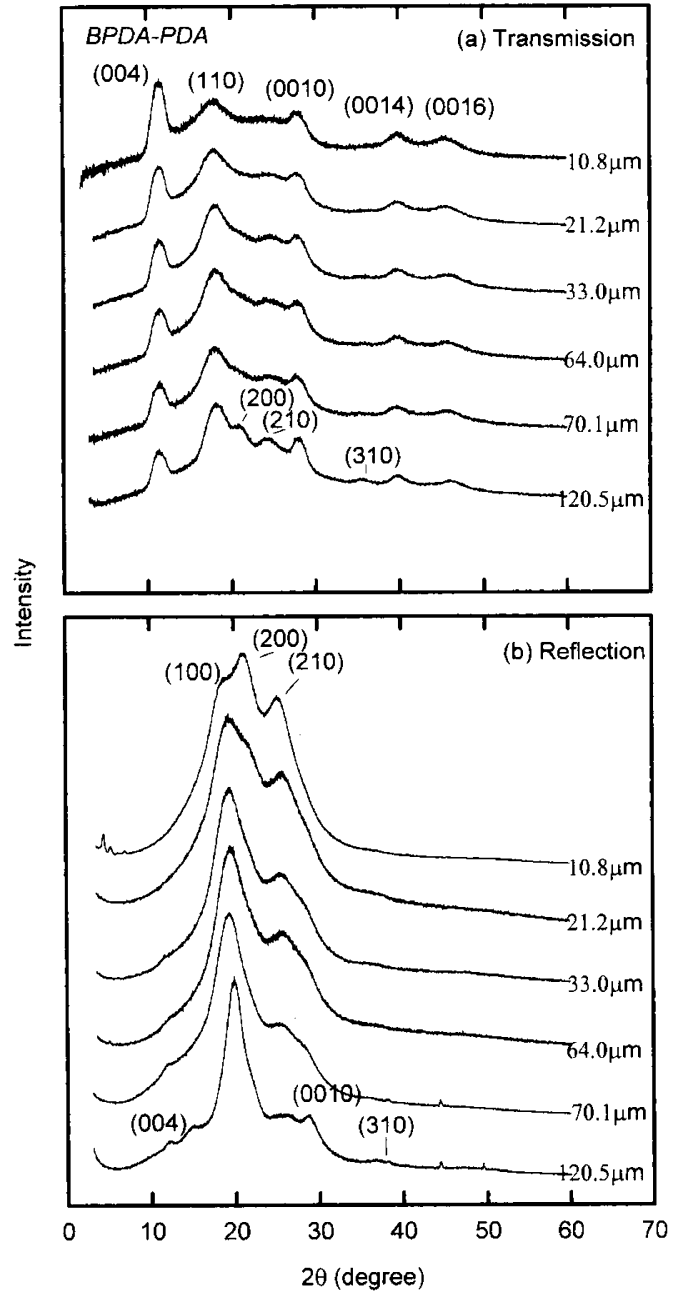

Figure 6. WAXD patterns of the BPDA-PDA polyimide films prepared with various thicknesses ; (a) transmission, (b) reflection.

width at half maximum of the $(00 l)$ peak. ${ }^{18,21}$ This means that the degree of molecular chain order along the chain axis for the thick polyimide films is relatively lower than for the thin films. However, the intensity of diffraction peak at $18^{\circ}$ was significantly enhanced in 
Table II. Change in $(0 O l)$ peak of polyimides with different film thicknesses

\begin{tabular}{|c|c|c|c|c|}
\hline & \multirow{2}{*}{$\frac{\text { Film thickness }}{\mu \mathrm{m}}$} & \multicolumn{2}{|c|}{ Mean intermolecular distances ${ }^{a}$} & \multirow{2}{*}{ Coherence length $^{\mathrm{d}}$} \\
\hline & & In-plane $\mathrm{e}^{\mathrm{b}} / \AA$ & Out-of-plane $/ \bar{\AA}$ & \\
\hline \multirow{6}{*}{ BPDA-PDA } & 10.8 & 4.94 & 4.78 & 122 \\
\hline & 21.2 & 4.94 & 4.74 & 115 \\
\hline & 33.0 & 4.92 & 4.64 & 111 \\
\hline & 64.1 & 4.90 & 4.63 & 108 \\
\hline & 70.1 & 4.90 & 4.63 & 106 \\
\hline & 120.5 & 4.88 & 4.60 & 105 \\
\hline \multirow{6}{*}{ PMDA-ODA } & 12.1 & 4.91 & 4.71 & 80 \\
\hline & 25.2 & 4.91 & 4.71 & 81 \\
\hline & 36.2 & 4.90 & 4.70 & 85 \\
\hline & 59.0 & 4.89 & 4.70 & 86 \\
\hline & 79.9 & 4.89 & 4.68 & 87 \\
\hline & 128.6 & 4.88 & 4.67 & 86 \\
\hline
\end{tabular}

${ }^{a}$ Estimated from the maximum of X-ray diffraction peaks corresponding to intermolecular spacing. ${ }^{\mathrm{b}}$ Calculated from the peak maximum of amorphous halos in transmission WAXD patterns. ${ }^{c}$ Calculated from the peak maximum of amorphous halos in reflection WAXD patterns. ${ }^{\mathrm{d}}$ Calculated from the peak maximum of $(00 l)$ peak in transmission WAXD patterns.

polyimide films with increasing thickness as shown in Figure 6 . This means that the lateral packing of chains combined with the amorphous halo is relatively enhanced for the thick films.

In contrast to transmission patterns, reflection patterns showed a higher intermolecular packing order in thick film than thin film. The (110) peak, which is representative of packing order, was relatively much sharper in shape and stronger in the intensity for thick film than thin film. For thick films $(>70 \mu \mathrm{m})$, two other diffraction peaks related to intermolecular packing were generated at $12^{\circ}$ and $28^{\circ}$ corresponding to $(004)$ and $(0010)$, respectively. Apparently, the presence of diffraction in reflection and transmission mode for thick $70 \mu \mathrm{m}$ film, not observed in thinner films, clearly indicates that the former is significantly more isotropic than the latter. This means that the chain anisotropy of BPDA-PDA polyimide films decreases with increasing thickness. The more segmental mobility of the polymer chains might be allowed due to more solvent content in thick film. The degree of molecular orientation in films would be lower because the effect of surface induced orientation ${ }^{22-25,27}$ was diminished as film thickness increased. This may lead to thicker films with more random structure with less chain anisotropy.

With these WAXD results, the degree of molecular inplane orientation in BPDA-PDA polyimide film is significantly degraded with increasing thickness in spite of slightly enhanced intermolecular packing order as shown in Figure 6. This indicates that the mainly reduced molecular in-plane orientation of BPDA-PDA might lead to higher residual stress with increasing film thickness. This suggests that the higher in-plane orientation depending upon film thickness might be one important factor of lower residual stress in BPDA-PDA polyimide. These show good agreement with residual stress behavior in BPDA-PDA polyimide films.

For PMDA-ODA films differing in thickness, morphological structure was investigated using WAXD as shown in Figure 7. The intensity of (002) diffraction peak for semiflexible PMDA-ODA corresponds to $d$ spacing of half the pitch of planar, zigzag chain conformation ${ }^{19}$ in transmission pattern. It was built up further in the range $>25 \mu \mathrm{m}$. This means the increase in the molecular in-plane orientation in thick film. The $(002)$

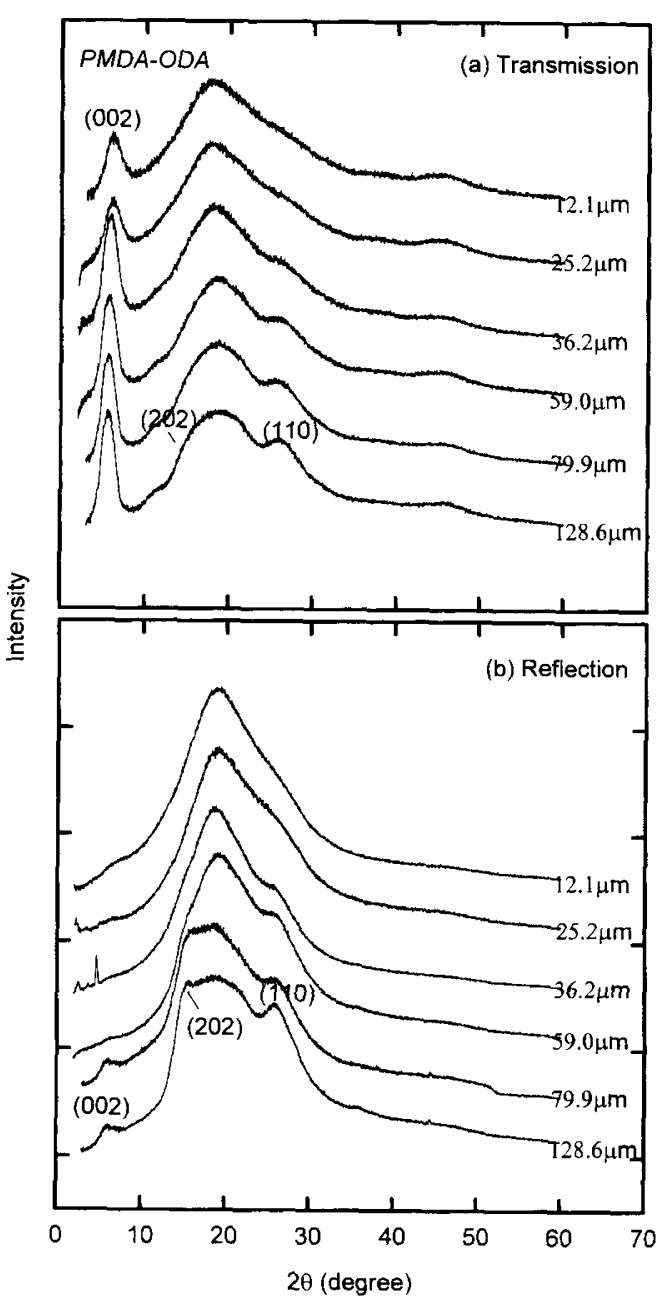

Figure 7. WAXD patterns of PMDA-ODA polyimide films prepared with various thicknesses ; (a) transmission, (b) reflection.

peak location for thicker film is at a lower $2 \theta$ corresponding to larger intermolecular $d$-spacing. The chain conformation of thicker film is thus more extended. Since the data are corrected for thickness, stronger intensity of the $(002)$ reflection in thicker film than thinner film indicates higher level of crystallinity in thick samples. This is consistent with earlier studies that thermal expansion decreases as thickness increases for 
PMDA-ODA adhered substrate. ${ }^{23}$ Increase in (002) peak with film thickness $>25 \mu \mathrm{m}$ thereafter leveled off at over $30-120 \mu \mathrm{m}$. The estimated coherence length for the (002) peak, which represents the degree of molecular chain order along the chain axis, was $80-87 \AA$, depending upon film thickness. The intensity of the diffraction peak at $18^{\circ}$ significantly diffused for the thick film. This corresponds to lateral packing of polymer chains in the film plane combined with the amorphous halo.

The reflection diffraction spectra of PMDA-ODA show similar trend in packing order like that of BPDA-PDA. The reflection pattern showed higher intermolecular packing order in thick film than thin film. Better chain conformational order in thicker compared to thinner film leads to better dimensional order. WAXD shown in Figure 7 in the intermediate angle range, for PMDA-ODA confirms that thicker film is more ordered than the thinner film. Here, two other diffraction peaks related to intermolecular packing were generated at $14^{\circ}$ and $28^{\circ}$. At $14^{\circ}$, the (202) reflection in the thicker film indicates that there is good order and registration along the $a$ axis. The thicker film has stronger (110) peak indicating ordered structure along $b$ axis. The absence of the reflections in the thinner film suggests that interchain registration of the chain are preferentially aligned in the film plane. The presence of $(002)$ in reflection and transmission mode for thick $80 \mu \mathrm{m}$ film, not observed in any of the thinner films, clearly indicates that the former is significantly more isotropic than the latter.

With increasing thickness, residual stress behavior in semiflexible PMDA-ODA smoothly increased in spite of enhancement of molecular in-plane orientation. This suggests that the effect of out-of plane orientation is more on smooth residual stress increase in semiflexible PMDA-ODA thick film. This confirms that the molecular anisotropy is more effective for low film stress than only molecular in-plane orientation. This indicates that the higher in-plane orientation and lower out-of plane orientation give the higher molecular anisotropy to result in lower film stress. However, the higher molecular anisotropy as well as lower residual stress are limited to only thin films regardless of chain chemistry. This was evident in pseudo rodlike BPDA-PDA polyimide.

In comparison of WAXD analysis for thin films of BPDA-PDA and PMDA-ODA, the (OOl) peaks appeared only on the transmission pattern. This means thin film has a high degree of polymer chain order along the chain axis. However for thick films (OOl) peaks appeared on both transmission and reflection patterns. This suggests that in thick film polymer chains are randomly aligned in the film plane and in the out-of film plane in thick film. Therefore, the higher residual stress and slope of cooling curve in thicker film might result mainly from less anisotropic orientation.

The degree of anisotropy variation in PMDA-ODA was significantly lower than that of the BPDA-PDA over the all range of thickness considered herein. The intensity of (004) diffraction peak in the transmission pattern of rigid BPDA-PDA significantly decreased with increasing thickness. However, the stronger intensity of the (002) diffraction in semiflexible PMDA-ODA thicker film than that in thinner film indicates higher crystallinity in the thick samples. This abnormal behavior might result from solvent contents and intrinsic chain mobility in PMDA-ODA polyimide films. The presence of a NMP greatly facilitates chain mobility and permit chain alignment during imidization process..$^{1-3}$ Because of the relatively high boiling point $\left(-202^{\circ} \mathrm{C}\right)$ and low vapor pressure of NMP, the trend to orient along $c$ axis for semiflexible PMDA-ODA may increase with film thickness. For complex formation of PAA and NMP, a prebaked poly(amic acid) specimen still contains typically $>20 \mathrm{wt}$ $\%$ of NMP, depending on the drying conditions. ${ }^{26}$ During thermal treatment more solvent would exist in the thick film at higher temperature than thin film. This indicates that with increasing thickness the temperature for the complete solvent evaporation might increase. By the same thermal treatment, thicker films are likely to retain molecular motion for a longer period of time for the solvent to diffuse out of the film. Prolonged plasticization by the solvent would increase the segmental mobility of polymer chains for molecular relaxation and allow thicker films more orientation along the $c$ axis for PMDA-ODA. In case of rigid BPDA-PDA, this effect might be less by higher chain rigidity and higher glass transition temperature $\left(\sim 430^{\circ} \mathrm{C}\right)$. These results due to chain mobility and solvent effect are in good accordance with the residual stress behavior of pseudo rodlike BPDA-PDA and semiflexible PMDA-ODA with increasing thickness.

Conclusively, molecular anisotropy is degraded to some extent with increasing film thickness regardless of rigid BPDA-PDA and semi-flexible PMDA-ODA. This means chain anisotropy is a critical factor in film stress. Specially, for planar BPDA-PDA films, in-plane orientation was significantly degraded compared to that for semi-flexible PMDA-ODA with its hinged structure. This suggests that higher residual stress in BPDA-PDA films might result from the less anisotropy induced by mainly weaker molecular in-plane orientation in thick film. However, in semiflexible PMDA-ODA film the degree of chain anisotropy decreased with film thickness in spite of increase in plane orientation. This indicates that slightly increased residual stress of semiflexible PMDAODA films results from less anisotropy induced by mainly solvent induced isotropic molecular packing in the out-of film plane.

\section{CONCLUSIONS}

Polyimides, the hinged structure PMDA-ODA and rigid planar structure BPDA-PDA, were investigated at various thicknesses from $8-130 \mu \mathrm{m}$. For fully cured BPDA-PDA polyimide at $400^{\circ} \mathrm{C}$, residual stress increased from $5.4 \mathrm{MPa}$ for $8.0 \mu \mathrm{m}$ thin film to $45.0 \mathrm{MPa}$ for $120.5 \mu \mathrm{m}$ thick film, respectively. For PMDA-ODA polyimide over the same thickness range of $8.0-129.0$ $\mu \mathrm{m}$, residual stress increased from $29.1 \mathrm{MPa}$ to 40.2 $\mathrm{MPa}$, respectively. The effect of thickness on the residual stress for the rigid structure BPDA-PDA was significant, but relatively not for the semi-flexible structure PMDA-ODA. This means that residual stress behavior is closely related to the morphological anisotropy of fully cured polyimide depending on thickness variation. Chain anisotropy for the rigid structure BPDA-PDA polyimide was significantly degraded with increasing 
thickness compared to semi-flexible PMDA-ODA polyimide. This suggests that the higher degree of molecular anisotropy in thin film leads to the lower residual stress of polyimide. This shows good agreement with the results of residual stress and morphology in polyimide thin films with thickness variation as shown in Figures 3,4 , 6 , and 7. Residual stress of polyimide films with thickness variation is thus closely related to chain rigidity and chain anisotropy associated with polyimide chemistry and morphological structure.

Acknowledgment. This work was supported by grant No. KOSEF 971-1102-007-2 from the Basic Research program of the Korea Science \& Engineering Foundation.

\section{REFERENCES}

1. K. L. Mittal, "Polyimides: Synthesis, Characterization and Application", Plenum., New York, N.Y., 1984.

2. D. Wilson, H. D. Stenzenberger, and P. M. Hergenrother., "Polyimides", Chapman \& Hall, New York, N.Y., 1990.

3. M. I. Bessonove, M. M. Koton, V. V. Kudryyavtsev, and L. A. Laius, "Polyimides : Thermally Stable Polymer", Consultants Bureau, New York, N.Y., 1987.

4. S. Numata, S. Ohara, J. Imaizumi, and N. Kinjo, Polym. J., 17, 981 (1985).

5. S. Numata and N. Kinjo, Polym. Eng. Sci., 28, 906 (1988).

6. J. Brekner and C. Feger, J. Polym. Sci., Part A: Polym. Chem., 25, 2005 (1987).

7. J. C. Coburn, M. T. Pottiger, S. C. Noe, and S. D. Senturia, Polymer, 32, 1271 (1993).

8. G. Elsner, J. Appl. Polym. Sci., 34, 815 (1987).

9. B. J. Han, C. C. Gryte, H. M. Tong, and C. Feger, ANTEC' 88
Proc., Society of plastics Engineers, Brookfield, CT, 34, 994 (1988).

10. C. L. Bauer and R. Farris, Polym. Eng. Sci., 29, 1107 (1989).

11. J. H. Jou and P. T. Huang, Macromolecules, 24, 3796 (1991).

12. J. H. Jou, P. T. Huang, H. C. Chen, and C. N. Liao, Polymer, 33, 967 (1992)

13. J. H. Jou, Y. L. Chang, and C. H. Liu, Macromolecules, 25, 5186 (1992)

14. M. Ree, C. W. Chu, and M. J. Goldberg, J. Appl. Phys., 75, 1410 (1994)

15. M. Ree, T. L. Nunes, K. J. Chen, and G. Czornyj, MRS Symposium Series, 227, 211 (1991).

16. S. P. Timoshenko, J. Opt. Soc. of America, 11, 223 (1926).

17. J. J. Wortman and R. A. Evans, J. Appl. Phys., 36, 136 (1965)

18. B. D. Cullity, "Elements of X-ray Diffraction", 2nd ed, Addison-Wesley Publ. Co., Reading, MA, 1978.

19. N. Takahashi, D. Y. Yoon, and W. Parrish, Macromolecules, 17, 2583 (1994)

20. H. M. Tong, C. K. Hu, C. Feger, and P. S. Ho, Polym. Eng. \& Sci., 26, 1213 (1970).

21. L. E. Alexander, "X-Ray Diffraction Method in Polymer Science", Robert E. Krieger Publ. Co., Huntington, N. Y., 1979.

22. H. Nomura, M. Eguchi, and M. Asano, J. Appl. Phys., 70, 7085 (1991).

23. M. T. Pottiger, J. C. Coburn, and J. R. Edman, J. Polym. Sci., Part B : Polym. Phys., 32, 825 (1994).

24. M. Ree, T. L. Nunes, G. Czornyj, and W. Volksen, Polymer, 33 1228 (1992).

25. H. Han, C. C. Gryte, and M. Ree, Polymer, 36, 1663 (1995).

26. T.-C. J. Hsu and Z.-L. Liu, J. Appl. Polym. Sci., 46, 1821 (1992).

27. L. Lin and S. A. Bidstrup, J. Appl. Polym. Sci., 49, 1277 (1993).

28. H. Chung, Y. Joe, and H. Han, Polym. J., 31, 700 (1999).

29. H. Chung, Y. Joe, and H. Han, J. Appl. Polym. Sci., 74, 3287 (1999). 